\title{
UNIVERSITY OF TRENTO
}

DEPARTMENT OF INFORMATION AND COMMUNICATION TECHNOLOGY

38050 Povo - Trento (Italy), Via Sommarive 14

http://www.dit.unitn.it

\author{
A THEORETICAL FRAMEWORK FOR UNSUPERVISED CHANGE \\ DETECTION BASED ON \\ Change Vector AnAlysis In Polar Domain \\ F. Bovolo, L. Bruzzone
}

March 2006

Technical Report DIT-06-019 



\title{
A Theoretical Framework for Unsupervised Change Detection Based on Change Vector Analysis in Polar Domain
}

\author{
F. Bovolo, IEEE Student Member, and L. Bruzzone, IEEE Senior Member
}

\begin{abstract}
This paper addresses unsupervised change detection by proposing a proper framework for a formal definition and a theoretical study of the change vector analysis (CVA) technique. This framework, which is based on the representation of the CVA in polar coordinates, aims at: i) introducing a set of formal definitions in the polar domain (which are linked to the properties of the data) for a better general description (and thus understanding) of the information present in spectral change vectors; ii) analyzing from a theoretical point of view the distributions of changed and unchanged pixels in the polar domain (also according to possible simplifying assumptions); iii) driving the implementation of proper pre-processing procedures to be applied to multitemporal images on the basis of the results of the theoretical study on the distributions; and iv) defining a solid background for the development of advanced and accurate automatic change-detection algorithms in the polar domain. The findings derived from the theoretical analysis on the statistical models of classes have been validated on real multispectral and multitemporal remote sensing images according to both qualitative and quantitative analyses. The results obtained confirm the interest of the proposed framework and the validity of the related theoretical analysis.
\end{abstract}

Index Terms-Change detection, change vector analysis, multitemporal images, unsupervised techniques, polar representation, spherical representation, statistical models, remote sensing. 


\section{INTRODUCTION}

Unsupervised change detection plays an important role in many application domains related to the exploitation of multitemporal remote sensing images. The availability of images acquired on the same geographical area by satellite sensors at different times makes it possible to identify and label possible changes occurred on the ground. In this context, in order to properly exploit the huge amount of data acquired by current remote sensing satellites, it is mandatory to develop effective unsupervised and automatic change-detection techniques.

Several unsupervised change-detection methodologies have been proposed in the literature [1][3]. Among them, a widely used technique is the change vector analysis (CVA). CVA is typically applied to multispectral images acquired by passive sensors, by considering more than one spectral channel in order to exploit all the available information about the considered event of change. However, usually CVA is used in an empirical way, without referring to a specific theoretical framework capable to properly and formally represent all the information contained in the spectral change vectors (SCVs) obtained by subtracting corresponding spectral bands of two images acquired at different dates. In addition, in the most of the applications, only the magnitude of the SCVs is exploited in order to identify changed pixels. Only in few applications also the direction of the vector is empirically used for deriving information on the kind of change occurred on the ground [4]-[13]. This lack of a formal framework and of a proper analysis of the statistics of data results in sub-optimal applications of the CVA or, in some cases, in a non-complete understanding of the richness of the information present in SCVs. This involves an incomplete exploitation of all the available information and/or the definition of change-detection algorithms which are not based on a solid theoretical background and on proper analysis procedures.

In order to address the aforementioned problems, in this paper we present a consistent theoretical framework for a proper representation, modeling and exploitation of the information present in the SCVs computed according to the CVA technique. The proposed framework and the related analysis 
are carried out in the context of a Polar representation of the CVA. In particular, the proposed novel contributions of this paper consist in: i) the introduction of formal definitions for a proper characterization of the information present in the polar CVA technique; ii) a theoretical analysis on the distributions of changed and unchanged pixels in the Polar domain under both general conditions and proper simplifying assumptions; iii) the introduction of proper guidelines for defining effective pre-processing strategies based on the expected properties of the theoretical distributions of changed and unchanged pixels; iv) the definition of a solid background for the development of advanced and accurate automatic change-detection algorithms in the Polar domain. A validation of the theoretical analysis, carried out on real multispectral and multitemporal remote sensing images, is reported.

The paper is organized into six sections. The next section introduces the change-detection problem and the formulation of the CVA technique in both Cartesian and Hyperspherical domains. Section III gives some basics on the models for joint conditional class distributions in both Cartesian and Polar coordinate systems. Section IV presents the proposed theoretical analysis on the models of marginal conditional distributions of magnitude and direction; furthermore, it proposes a critical analysis on the importance and the effects of image pre-processing procedures (e.g., radiometric corrections, coregistration, etc.) on the data distributions. The validation of the proposed theoretical analysis, carried out on real multitemporal and multispectral remote sensing data, is reported in section V. Finally, Section VI discusses the obtained results and draws the conclusions of this paper.

\section{Proposed Polar REPRESENTATION FrameWORK FOR CVA}

\section{A. Background and CVA Formulation}

Let us consider two coregistered multispectral images, $\mathbf{X}_{1}$ and $\mathbf{X}_{2}$, of size $I \cdot J$, acquired over the same area at different times $t_{1}$ and $t_{2}$. Let $X_{1}$ and $X_{2}$ be two multidimensional random variables that represent the statistical distributions of pixels in images $\mathbf{X}_{1}$ and $\mathbf{X}_{2}$, respectively. Let $X_{b, t}$ be the 
random variable representing the $b$-th component of the multispectral image $\mathbf{x}_{t}(t=1,2)$ in the considered feature space. Let $\boldsymbol{\Omega}=\left\{\omega_{n}, \boldsymbol{\Omega}_{c}\right\}$ be the set of classes of unchanged and changed pixels to be identified. In greater detail, $\omega_{n}$ represents the class of unchanged pixels and $\boldsymbol{\Omega}_{c}=\left\{\omega_{c_{1}}, \ldots, \omega_{c_{\mathrm{K}}}\right\}$ the set of the $K$ possible classes (kinds) of change occurred in the considered area.

The first step of the most widely used change-detection techniques presented in the literature performs comparison between the two considered images according to a proper operator [1]. When dealing with multispectral images, the most widely used comparison operator is the difference. In many cases difference is applied to a $n$-dimensional feature space in order to give as input to the change-detection process all the relevant spectral information. This technique is known as change vector analysis (CVA) [1],[5] and has been successfully used in many different application domains [4]-[13] ${ }^{1}$. CVA firstly computes a multispectral difference image $\left(\mathbf{X}_{\boldsymbol{D}}\right)$ subtracting the spectral feature vectors associated with each corresponding spatial position in the two considered images $\mathbf{X}_{1}$ and $\mathbf{X}_{2}$. Let $X_{D}$ be the multidimensional random variable representing the spectral change vectors $(\mathrm{SCVs})$ in the difference image obtained as follows [1]:

$$
\mathbf{X}_{D}=\mathbf{X}_{2}-\mathbf{X}_{1}
$$

Each SCV is usually implicitly represented in Polar coordinates with its magnitude and direction. Although the direction of the SCVs is rich of information (e.g., on the kind of changes occurred on the ground and on the distribution of registration noise), in the most of the applications it is not considered. Among the few studies reported in the literature where magnitude and direction expressed as cosine functions are considered together for change detection, we recall [5]-[13]. In 1980, Malila [5] first formulated the concept of change vector and then used both magnitude and direction in a two dimensional space for identifying changes due to plants clearcut and regrowth in the northern Idaho (U.S.) forest. In [5]-[8] the direction variable was subdivided in a fixed number of sectors, each of them corresponding to positive or negative changes in one of the $B$ considered 
features (i.e., spectral channels or linear combinations of them, like Tasseled-Cap transformation). This kind of quantization leads to the definition of a maximum of $2^{B}$ sectors and hence of types of changes. The major drawback of this approach is that different kinds of changes could assume the same sector code. In [9], the CVA sector coding approach was extended to the solution of multivariate, full-dimensional and also multi-interval problems (i.e., applications involving more than two acquisition dates). In [10], Allen and Kupfer introduced in the CVA technique the use of direction cosines for the description of SCV directions. They applied a hierarchical linear discriminant analysis for testing predictive power of magnitude and vector angles in solving changedetection problems. Direction cosines were used also in [11] and [12]. In these works authors firstly identified changed pixels on the basis of magnitude values, then image classification algorithms were applied to direction cosines for discriminating the different kinds of change. In [13], authors defined a modified CVA (mCVA) technique where Polar coordinates are transformed back into a Cartesian coordinate system to overcome discontinuity between 0 and $2 \pi$ and different kinds of changes are then detected applying either supervised or unsupervised clustering algorithms in the defined domain. A different approach to the use of the direction information has been presented in [4], where the authors proposed a method for estimating and reducing the registration noise. The method is based on a joint exploitation of the magnitude and direction components. However, the most of the analyses reported in the literature have been carried out in an empirical way as well as without a rigorous statistical information class characterization and without referring to a proper theoretical framework for completely understanding and processing the information present in SCVs.

In this paper, in order to fill the aforementioned gaps in the current CVA formulation and to provide a solid background to improve the efficiency of the CVA technique in real data analysis, we propose a rigorous framework characterized by proper formal definitions for data representation in a

1 The particular case of working with a single feature reduces the CVA to the univariate image differencing technique [1]. 
Polar coordinate system. In addition, we peresent a theoretical analysis of the data distributions in the Polar domain and derive from this analysis interesting properties of the SCVs, which are very useful for driving both the pre-processing procedure to be applied to multitemporal data and the definition of effective change-detection algorithms.

\section{B. General Framework for Hyperspherical Representation}

Given a $B$-dimensional feature space, the SCV associated with a pixel of the analyzed scene can be described with its magnitude value and $B-1$ directions. In this work, we propose to represent the properties of the SCVs, instead of using a Cartesian coordinate system, by plotting SCVs in a $B$ dimensional Hyperspherical coordinate system ${ }^{2}$. Thus the multidimensional random variable $X_{D}$ can be represented with a random variable $\rho$ that models the statistical distribution of the change vector magnitude and $B-1$ random variables $\left[\vartheta_{1}, \vartheta_{2}, \ldots, \vartheta_{B-1}\right]$ that represent the distribution of the change vector angular coordinates. It is worth noting that changing the coordinate system has a dramatic impact on the statistical distributions of the considered classes. This aspect will be analyzed in the next section.

Let $X_{1, D}, \ldots, X_{B, D}$ be the random variables representing the distributions of SCVs along the $B$ dimensions (spectral channels) of the considered Cartesian coordinate system; then, the relations between the random variables modeling SCVs in the Cartesian and the Hyperspherical coordinates are the following:

$$
\begin{aligned}
& X_{1, D}=\rho \cos \vartheta_{1} \\
& X_{2, D}=\rho \operatorname{sen} \vartheta_{1} \cos \vartheta_{2} \\
& X_{3, D}=\rho \operatorname{sen} \vartheta_{1} \operatorname{sen} \vartheta_{2} \cos \vartheta_{3} \\
& \ldots . \\
& X_{B, D}=\rho \operatorname{sen} \vartheta_{1} \ldots \operatorname{sen} \vartheta_{B-2} \cos \vartheta_{B-1}
\end{aligned}
$$

where $\vartheta_{1} \in[0,2 \pi)$ and $\vartheta_{2}, \ldots, \vartheta_{B-1} \in[0, \pi]$.

In the following, for simplicity, we will assume that the CVA technique is applied only to two

2 In the particular case of $B=2$ the Hyperspherical coordinate system is said Polar coordinate system. 
spectral channels of the considered multitemporal images, i.e., that a two-dimensional coordinate system is sufficient to completely describe SCVs. However, the analysis can be generalized to the case of more spectral channels by considering more direction contributions for describing each $\mathrm{SCV}$. It is worth noting that the assumption of working with a couple of spectral channels is reasonable in many change detection problems [14]-[16]. This choice is often due to the need of isolating the most informative features with respect to the specific considered problem without including noisy and misleading spectral channels in the analysis. In the above assumption, in the Cartesian coordinate system only random variables $X_{1, D}$ and $X_{2, D}$ are necessary to describe SCVs, whereas in the Polar coordinate system random variables representing the magnitude $\rho$ and one direction $\vartheta\left(\equiv \vartheta_{1}\right)$ are required for each SCV. The relation between Cartesian and Polar representation of the difference image is as follows:

$$
\left\{\begin{array}{l}
\vartheta=\tan ^{-1}\left(\frac{X_{1, D}}{X_{2, D}}\right) \\
\rho=\sqrt{\left(X_{1, D}\right)^{2}+\left(X_{2, D}\right)^{2}} .
\end{array}\right.
$$

\section{Proposed Polar framework for the CVA technique: definitions}

In this section we propose a rigorous characterization of the Polar framework for the CVA technique. First of all, observe that in the Polar representation, all the change vectors of a given scene are included in a domain $D$ defined as (see Fig. 1):

$$
D=\left\{\rho \in\left[0, \rho_{\max }\right] \text { and } \vartheta \in[0,2 \pi)\right\}
$$

where $\rho_{\max }$ is the maximum value assumed by the magnitude on the considered image, i.e.,

$$
\rho_{\max }=\max \left\{\sqrt{\left(X_{1, D}\right)^{2}+\left(X_{2, D}\right)^{2}}\right\}
$$

According to the given definition of $D$, in order to establish a clear framework for CVA, we propose to identify different regions in the magnitude-direction domain for pointing out the information present in SCVs. From the definition in (4) and following consideration in [16], we expect that unchanged pixels have magnitude close to zero (often not exactly zero due to the presence of noise 
components), while changed pixels have magnitude far from zero. Consequently it is possible to identify two different regions associated with: i) unchanged and ii) changed pixels. Thus the Polar domain can be split into two parts: i) circle $C_{n}$ of no-changed pixels; and ii) annulus $A_{c}$ of changed pixels. This can be done according to the optimal (in the sense of the theoretical Bayesian decision theory) threshold value $T$ that separates pixels belonging to $\omega_{n}$ from pixel belonging to $\boldsymbol{\Omega}_{c}$ (dark gray and light gray areas in Fig. 1, respectively).

Definition 1: the Circle of unchanged pixels $C_{n}$ is defined as

$$
C_{n}=\{\rho, \vartheta: 0 \leq \rho<T \text { and } 0 \leq \vartheta<2 \pi\}
$$

$C_{n}$ can be represented in the Polar domain as a circle with radius $T$. From this definition we can state that for the generic pixel (spatial position) $(i, j)$, it holds that:

$$
(i, j) \in \omega_{n} \Leftrightarrow(i, j) \in C_{n}
$$

or in other words:

$$
(i, j) \in \omega_{n} \Leftrightarrow \rho(i, j)<T
$$

This means that all the unchanged pixels satisfy (7) [or equivalently (8)] and are included in $C_{n}$.

Definition 2: the Annulus of changed pixels $A_{c}$ is defined as:

$$
A_{c}=\left\{\rho, \vartheta: T \leq \rho \leq \rho_{\max } \text { and } 0 \leq \vartheta<2 \pi\right\} .
$$

$A_{c}$ can be represented in the Polar domain as a ring with inner radius $T$ and outer radius $\rho_{\max }$. From this definition we can state that for the generic spatial position $(i, j)$, it holds that:

$$
(i, j) \in \Omega_{c} \Leftrightarrow(i, j) \in A_{c}
$$

or in other words:

$$
(i, j) \in \Omega_{c} \Leftrightarrow T \leq \rho(i, j) \leq \rho_{\max }
$$

This means that all the changed pixels satisfy (10) [or equivalently (11)] and are included in $A_{c}$. According to the above definitions, the Polar domain can be described as the union of $A_{c}$ and $C_{n}$, 
i.e.,

$$
D=A_{c} \cup C_{n}
$$

In real applications, often the pixels with magnitude close to the optimal (in the sense of the theoretical Bayesian decision theory) threshold value $T$ can not be accurately labeled according to a simple thresholding procedure due to the intrinsic uncertainty present in the data. In these cases, by taking into account that the spatial autocorrelation function of the images is not impulsive (i.e., pixels are spatially correlated $)^{3}$, it is possible to support the decision process according to a contextsensitive analysis of the investigated pixel [16]. This analysis is aimed at exploiting the spatial correlation as an additional information source in the decision process. In order to model and represent this uncertainty in the proposed framework, we can define a third (optional) region, i.e., the annulus $A_{u}$ of uncertain pixels. In Fig. 1 this region is depicted as a hatched annulus that partially overlaps both $C_{n}$ and $A_{c}$.

Definition 3: the Annulus $A_{u}$ of uncertain pixels is defined as:

$$
A_{u}=\{\rho, \vartheta: T-\alpha \leq \rho \leq T+\alpha \text { and } 0 \leq \vartheta<2 \pi\}
$$

where $\alpha$ is a parameter that defines the margin around $T$ in which pixels cannot be easily identified as either changed or unchanged. $A_{u}$ can be represented in the Polar domain as a ring with inner radius $T$ - $\alpha$ and outer radius $T+\alpha$. From this definition, we can state that:

$$
(i, j) \in A_{u} \Leftrightarrow T-\alpha \leq \rho(i, j) \leq T+\alpha
$$

This means that all the uncertain pixels satisfying (14) are included in $A_{u}$. The use of this definition depends on the specific data analysis strategy (if no contextual information is considered, it is assumed that $\alpha=0$ and consequently $A_{u}=\varnothing$ ).

The previous definitions have been based on the values of the magnitude, independently on the 
direction variable. A further important definition is related to sectors in the Polar domain, which are mainly related to the direction of the change vectors and therefore to the kinds of change occurred on the ground.

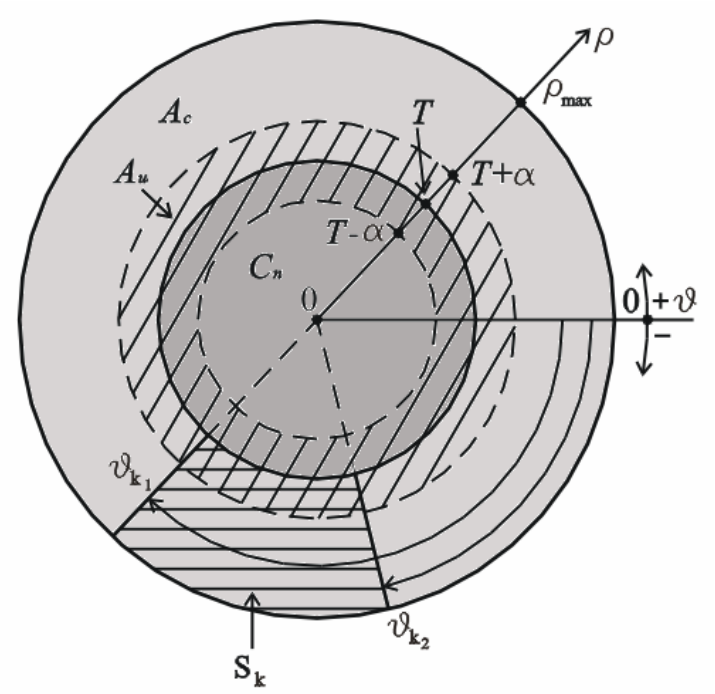

Figure 1 Representation of the regions of interest for the CVA technique in the Polar coordinate system.

Definition 4: the Annular sector $S_{k}$ of change $\omega_{c_{k}} \in \mathbf{\Omega}_{c}$ is defined as:

$$
S_{k}=\left\{\rho, \vartheta: \rho \geq T \text { and } \vartheta_{k_{1}} \leq \vartheta<\vartheta_{k_{2}}, 0 \leq \vartheta_{k_{1}}<\vartheta_{k_{2}}<2 \pi\right\}
$$

$S_{k}$ can be represented in the Polar domain as a sector of change within the annulus of changed pixels [see (9)] and bounded from two angular thresholds $\vartheta_{k_{1}}$ and $\vartheta_{k_{2}}$ (see Fig. 1). We expect that pixels that belong to the same kind of change are included in the same sector ${ }^{4}$. In the Polar coordinate system, two angular coordinates identify two sectors: i) a convex sector, and ii) a concave sector. As it is reasonable to expect that pixels belonging to the same change class have a low variance, generally the sector we are interested to is the convex one. It is worth noting that this condition is no longer satisfied if the convex sector covers the discontinuity between 0 and $2 \pi$. In this case the variance of the changed pixels is high and the relation between the two angular coordinates is inverted, i.e., $\vartheta_{k_{1}}>\vartheta_{k_{2}}$; thus, the definition of $S_{k}$ becomes:

3 This is true under the reasonable assumption that the geometrical resolution of the considered multispectral sensor is proper for the analyzed scene. 


$$
S_{k}=\left\{\rho, \vartheta: \rho \geq T \text { and } \vartheta_{k_{1}} \leq \vartheta<2 \pi \cup 0 \leq \vartheta<\vartheta_{k_{2}}, \quad 0 \leq \vartheta_{k_{1}}<\vartheta_{k_{2}}<2 \pi\right\}
$$

Fig. 1 depicts an example of annular sector as a hatched sector of annulus that overlaps region $A_{c}$ between angular coordinates $\vartheta_{k_{1}}$ and $\vartheta_{k_{2}}$.

\section{ANALYSIS OF THE JOINT CONDITIONAL DistribUtions OF ClASSES}

The definition of the different regions of interest in the Polar domain allows a better representation of the change-detection problem and drives to the analysis of another important problem that concerns the expected distribution of classes of interest in the change-detection problem.

\section{A. Class distributions in the Cartesian domain}

As known from the literature [17], the statistical distribution of natural classes in images acquired by multispectral passive sensors can be considered approximately Gaussian. Thus, both multidimensional random variables $X_{1}$ and $X_{2}$ can be modeled as a mixture of multidimensional Gaussian distributions in the Cartesian domain. As $X_{D}$ is obtained subtracting $X_{1}$ from $X_{2}$, its distribution can be also reasonably represented as a mixture of multidimensional Gaussian distributions, each of them associated with a class $\omega_{i}, \omega_{i} \in \mathbf{\Omega}=\left\{\omega_{n}, \boldsymbol{\Omega}_{c}\right\}=\left\{\omega_{n}, \omega_{c_{1}}, \ldots, \omega_{c_{K}}\right\}$ :

$$
p\left(X_{D}\right)=P\left(X_{D} \mid \omega_{n}\right)+P\left(X_{D} \mid \mathbf{\Omega}_{c}\right)=P\left(X_{D} \mid \omega_{n}\right)+\sum_{k=1}^{K} P\left(X_{D} \mid \omega_{c_{k}}\right)
$$

where $P\left(X_{D} \mid \omega_{i}\right)$ is a normal conditional density that models the distribution of the class $\omega_{i}$ in the multivariate difference image. As classes in $X_{D}$ can be approximated as jointly Gaussian distributed, it is possible to show that all the components $X_{b, D}$, obtained subtracting corresponding spectral bands $(b=1,2)$, are also a mixture of normally distributed random variables. This consideration and the assumption in (17) are the starting point for analyzing the statistical distributions of the no-change class and of the $K$ classes of change in the Polar domain.

4 It is worth noting that sectors associated with different kinds of change are not overlapped. 


\section{B. Class distributions in the Polar domain}

From (17), it can be seen that the analytical expression of class distributions in the Polar domain can be obtained by computing the joint conditional probability density functions of the magnitude and direction of SCVs [see (3)] of the 2-dimensional random variable $X_{D}$. A simplifying hypothesis is to consider features $X_{1, D}$ and $X_{2, D}$ as independent (see section IV.C for a detailed discussion on this hypothesis). Under this assumption, the distribution of the class $\omega_{i}\left(\omega_{i} \in \Omega\right)$ in a Cartesian coordinate system can be written as the product of the two marginal densities $P\left(X_{b, D} \mid \omega_{i}\right)$ of the class $\omega_{i}(b=1,2)$, i.e.,

$$
P\left(X_{D} \mid \omega_{i}\right)=\frac{1}{2 \pi \sigma_{1, i} \sigma_{2, i}} \exp \left[-\frac{\left(X_{1, D}-\mu_{1, i}\right)^{2}}{2 \sigma_{1, i}^{2}}-\frac{\left(X_{2, D}-\mu_{2, i}\right)^{2}}{2 \sigma_{2, i}^{2}}\right]
$$

where $\mu_{b, i}$ and $\sigma_{b, i}$ are the mean values and the standard deviations, respectively, of the Gaussian distributed marginal density of class $\omega_{i}$ over the $b$-th considered feature $(b=1,2)$. Applying the transformation from Cartesian to Polar coordinate system, the joint conditional distribution can be written as:

$$
P\left(\rho, \vartheta \mid \omega_{i}\right)=\frac{1}{2 \pi \sigma_{1, i} \sigma_{2, i}} \exp \left[-\frac{\left(\rho \cos \vartheta-\mu_{1, i}\right)^{2}}{2 \sigma_{1, i}^{2}}-\frac{\left(\rho \operatorname{sen} \vartheta-\mu_{2, i}\right)^{2}}{2 \sigma_{2, i}^{2}}\right]
$$

According to this general equation (which is the starting point for the statistical analysis reported in the next section) we can define the quantities typically used for evaluating the performance of change-detection algorithms (i.e., false alarms and missed alarms) in the context of the proposed framework. Let us define the following decision regions:

i) the region of changed pixels $\left(R_{c}\right)$ that corresponds to the union of all identified nonoverlapping annular sectors $S_{k}(k=1, \ldots, K): R_{c}=\bigcup_{k=1}^{K} S_{k}$;

ii) the region of no-changed pixels $\left(R_{n}\right)$ that corresponds to the union of circle of no-change $C_{n}$ and the region $A_{c}-\bigcup_{k=1}^{K} S_{k}$ complementary to the region of changed pixels with respect to 


$$
A_{c} \text {, i.e. } R_{n}=C_{n} \cup\left\{A_{c}-\bigcup_{k=1}^{K} S_{k}\right\}
$$

Given these decision regions and the joint conditional distributions for the classes of change and nochange in (19), it is possible to analytically define the false and missed alarms.

False alarms occur when unchanged pixels are identified as changed. The probability of this kind of error $P_{f}$ can be written as the integral of the joint conditional probability density function given the class of no-change over the region of changed pixels, i.e.

$$
P_{f}=\int_{R_{c}} P\left(\rho, \vartheta \mid \omega_{n}\right) d \rho d \vartheta
$$

Missed alarms occur when changed pixels are identified as unchanged. The probability of this kind of error $P_{m}$ can be written as the sum of the $K$ integrals (one for each class of change $\omega_{c_{k}}$ ) of the joint conditional probability density function given the class $\omega_{c_{k}}$ of change over the region associated to unchanged pixels, i.e.

$$
P_{m}=\sum_{k=1}^{K} \int_{R_{n}} P\left(\rho, \vartheta \mid \omega_{c_{k}}\right) d \rho d \vartheta
$$

\section{AnAlysis of the Marginal CONDitional Distributions of Magnitude AND DiRECTION}

The joint conditional probability in (19) is too general to be efficiently used in solving changedetection problems. A more suitable way to approach the problem is to compute the marginal conditional densities of the magnitude $\rho$ and the direction $\vartheta$. Starting from (19), these two densities can be computed for each class $\omega_{i}$ by integrating (19) over the range of $\vartheta$ and $\rho$, respectively.

Let us first consider the marginal conditional density of the magnitude $p\left(\rho \mid \omega_{i}\right)$. Integrating (19) over the range of $\vartheta$ leads to the following equation:

$$
p\left(\rho \mid \omega_{i}\right)=\frac{\rho}{2 \pi \sigma_{1, i} \sigma_{2, i}} \int_{0}^{2 \pi} \exp \left[-\frac{\left(\rho \cos \vartheta-\mu_{1, i}\right)^{2}}{2 \sigma_{1, i}^{2}}-\frac{\left(\rho \operatorname{sen} \vartheta-\mu_{2, i}\right)^{2}}{2 \sigma_{2, i}^{2}}\right] \mathrm{d} \vartheta
$$

This integral can not be expressed in a closed form, but it can be reduced to an infinite series of 
Bessel functions. By following [18], it is possible to show that $p\left(\rho \mid \omega_{i}\right)$ can be written as:

$$
p\left(\rho \mid \omega_{i}\right)=\frac{\rho}{\sigma_{1, i} \sigma_{2, i}} \exp (-D) \sum_{p=0}^{\infty}(-1)^{p} \varepsilon_{p} I_{p}(P) I_{2 p}\left(\sqrt{Q^{2}+R^{2}}\right) \cos \left(2 p \arctan \frac{R}{Q}\right), \rho \geq 0
$$

where $I_{p}(z)$ is the $p$-th order modified Bessel function of the first kind defined as:

$$
\mathrm{I}_{p}(z)=\frac{1}{2 \pi} \int_{C}^{C+2 \pi} \exp (-z \cos (u)+i p u) d u
$$

where $C$ is a constant, $i$ is the imaginary unit, and $D, P, Q$ and $R$ are defined as follows:

$$
D=\frac{\mu_{1, i}^{2}}{2 \sigma_{1, i}^{2}}+\frac{\mu_{2, i}^{2}+\rho^{2}}{2 \sigma_{2, i}^{2}}+\frac{\sigma_{2, i}^{2}-\sigma_{1, i}^{2}}{4 \sigma_{1, i}^{2} \sigma_{2, i}^{2}} \rho^{2}, \quad P=\frac{\sigma_{2, i}^{2}-\sigma_{1, i}^{2}}{4 \sigma_{1, i}^{2} \sigma_{2, i}^{2}} \rho^{2}, \quad Q=\frac{\mu_{1, i} \rho}{\sigma_{1, i}^{2}}, \quad R=\frac{\mu_{2, i} \rho}{\sigma_{2, i}^{2}} .
$$

The marginal conditional density of the direction $p\left(\vartheta \mid \omega_{i}\right)$ can be obtained by integrating (19) over the range of $\rho$. The integral can be written in a closed form. It is possible to prove that after some handling we obtain:

$$
\begin{gathered}
p\left(\vartheta \mid \omega_{i}\right)=\frac{\left(1+\tan ^{2}(\vartheta)\right)}{4 \pi \sqrt{\left(\sigma_{1, i}^{2} \tan ^{2}(\vartheta)+\sigma_{2, i}^{2}\right)^{3}}}\left\{\operatorname { e x p } ( - \frac { \mu _ { 2 , i } ^ { 2 } \sigma _ { 1 , i } ^ { 2 } + \mu _ { 1 , i } ^ { 2 } \sigma _ { 2 , i } ^ { 2 } } { 2 \sigma _ { 1 , i } ^ { 2 } \sigma _ { 2 , i } ^ { 2 } } ) \left[\sqrt{2 \pi} \exp \left(-\frac{\left(\mu_{2, i} \sigma_{1, i}^{2} \tan (\vartheta)+\mu_{1, i} \sigma_{2, i}^{2}\right)^{2}}{2 \sigma_{1, i}^{2} \sigma_{2, i}^{2}\left(\sigma_{1, i}^{2} \tan ^{2}(\vartheta)+\sigma_{2, i}^{2}\right)}\right)\right.\right. \\
\left.\left.\left(\mu_{2, i} \sigma_{1, i}^{2} \tan (\vartheta)+\mu_{1, i} \sigma_{2, i}^{2}\right)\left[1+\operatorname{erf}\left(\frac{\mu_{2, i} \sigma_{1, i}^{2} \tan (\vartheta)+\mu_{1, i} \sigma_{2, i}^{2}}{\sigma_{1, i} \sigma_{2, i} \sqrt{2\left(\sigma_{1, i}^{2} \tan ^{2}(\vartheta)+\sigma_{2, i}^{2}\right)}}\right)\right]+2 \sigma_{1, i} \sigma_{2, i} \sqrt{\left(\sigma_{1, i}^{2} \tan ^{2}(\vartheta)+\sigma_{2, i}^{2}\right)}\right]\right\}, \\
\vartheta \in[0,2 \pi) .
\end{gathered}
$$

Figs. 3 and 4 show examples of the behaviors of the magnitude and direction marginal conditional densities versus the mean values $\left(\mu_{1, i}, \mu_{2, i}\right)$ and the standard deviations $\left(\sigma_{1, i}, \sigma_{2, i}\right)$, respectively, of the class $\omega_{i}$ characterized by a Gaussian distribution in the Cartesian coordinate system. It is worth noting that the periodicity of the direction distribution depends on the period of the tangent function; in real applications the proper maximum should be selected according to the data distribution.

As can be seen, (23) and (26) represent two complex mathematical expressions. In real applications, usually additional hypotheses can be made in order to simplify the analytical expressions of the probability density functions. In the change-detection problem, different assumptions can be formulated for the classes of changed and unchanged pixels. In the following, 
the cases related to the two classes of interest will be addressed separately and in greater detail.

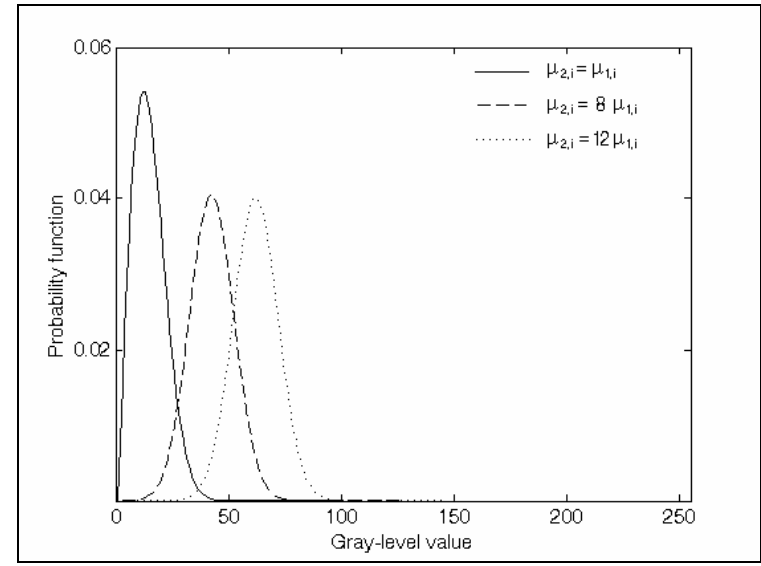

(a)

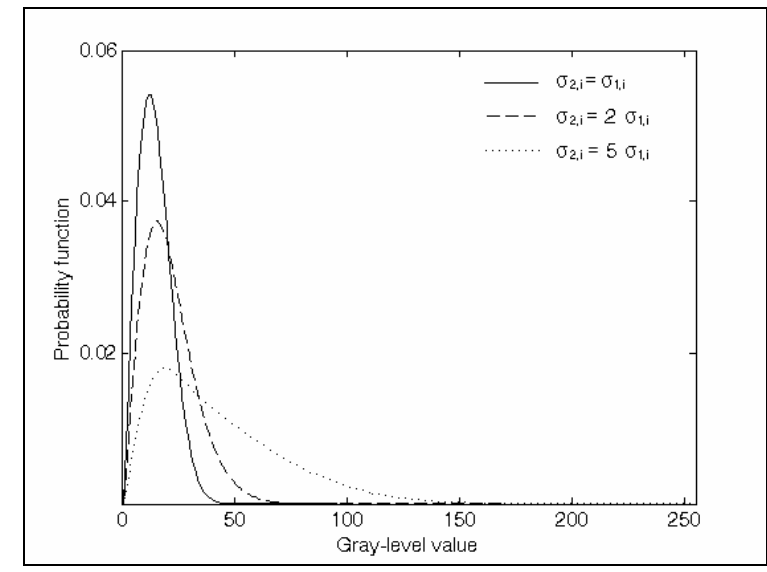

(b)

Figure 2 Examples of conditional distributions of the magnitude: (a) with respect to different values of $\mu_{2, i}$ $\left(\mu_{1, i}=5, \sigma_{1, i}=\sigma_{2, i}=10\right)$; and (b) with respect to different values of $\sigma_{2, i}\left(\mu_{1, i}=\mu_{2, i}=5\right.$ and $\sigma_{1, i}$ $=10)$.

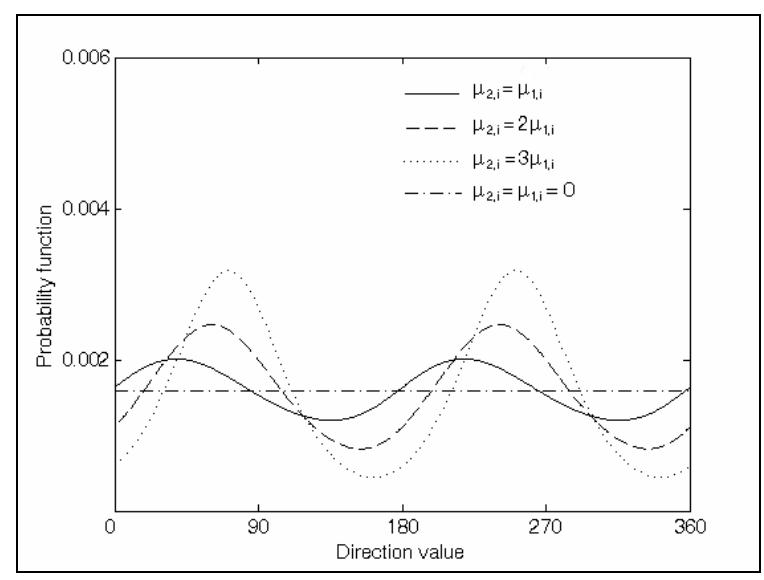

(a)

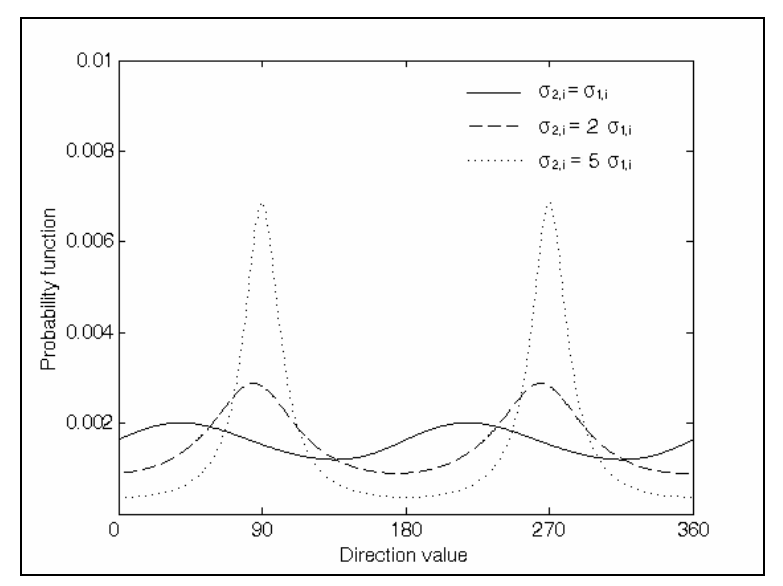

(b)

Figure 3 Examples of conditional distributions of the direction: (a) with respect to different values of $\mu_{2, i}$ ( $\mu_{1, i}$ $\left.=5, \sigma_{1, i}=\sigma_{2, i}=10\right)$ and in the particular case of $\mu_{1, i}=\mu_{2, i}=0$ that leads to the Uniform distribution; and (b) with respect to different values of $\sigma_{2, i}\left(\mu_{1, i}=\mu_{2, i}=5\right.$ and $\left.\sigma_{1, i}=10\right)$.

\section{A. Statistical models for the class of unchanged pixels}

As stated in section III.B, we assume that images $\mathbf{X}_{\mathbf{1}}$ and $\mathbf{X}_{\mathbf{2}}$ have been coregistered [19],[20] and that possible differences in the light and atmospheric conditions at the two times have been 
corrected $[21]^{5}$. Under these hypotheses, we can reasonably assume that in unchanged areas natural classes do not significantly change their distributions between the two acquisition dates. This simplifies the computation of distributions in the Polar domain as we can write:

$$
\begin{gathered}
\mu_{1, \omega_{n}} \cong \mu_{2, \omega_{n}} \approx 0 \\
\sigma_{1, \omega_{n}} \cong \sigma_{2, \omega_{n}}=\sigma_{\omega_{n}} .
\end{gathered}
$$

Substituting both expressions (27) and (28) into (22) and solving the integral, we get for the magnitude random variable the following probability density function:

$$
p\left(\rho \mid \omega_{n}\right)=\frac{\rho}{\sigma_{\omega_{n}}^{2}} \exp \left(-\frac{\rho^{2}}{2 \sigma_{\omega_{n}}^{2}}\right), \rho \geq 0
$$

which is commonly known as the Rayleigh distribution.

Concerning the statistical distribution of the direction variable for the class of unchanged pixels, it can be obtained substituting (27) and (28) into (26) i.e.,

$$
p\left(\vartheta \mid \omega_{n}\right)=\frac{1}{2 \pi}, \quad \vartheta \in[0,2 \pi)
$$

This means that the statistical distribution of the direction is Uniform within $[0,2 \pi)$.

\section{B. Statistical models for the classes of changed pixels}

The analytical study of the distribution of the generic class $\omega_{c_{k}}, \omega_{c_{k}} \in \Omega_{c}$, (for simplicity of notation in the following $\omega_{c_{k}}$ will be indicated as $\omega_{k}$ ) of changed pixels is more complex than the one carried out for the class of unchanged pixels. In this case, assumption (27) is no further valid, as changes in land-cover types modify the mean values of the natural classes in different ways in different spectral channels (this depends on the kind of change). This leads to the following condition:

$$
\mu_{1, \omega_{k}} \neq \mu_{2, \omega_{k}} \neq 0
$$

It is worth noting that if $\mu_{1, \omega_{k}}=\mu_{2, \omega_{k}}$ or they are both equal to 0 the analysis of the distribution is

5 This assumption will be discussed in section IV.C. 
simplified. In order to further simplify the computation of the magnitude and direction statistical distributions, we can assume that:

$$
\sigma_{1, \omega_{k}} \approx \sigma_{2, \omega_{k}}=\sigma_{\omega_{k}}
$$

In some applications this assumption is reasonable, but its validity should be verified for any specific case considered ${ }^{6}$. Thus, rewriting (22) according to (31) and (32) and solving the integral, it is possible to show that the random variable representing the magnitude is Ricean distributed, with probability density function given by:

$$
p\left(\rho \mid \omega_{k}\right)=\frac{\rho}{\sigma_{\omega_{k}}^{2}} \exp \left(-\frac{\rho^{2}+M_{\omega_{k}}^{2}}{2 \sigma_{\omega_{k}}^{2}}\right) \mathrm{I}_{0}\left(\frac{\rho M_{\omega_{k}}}{\sigma_{\omega_{k}}^{2}}\right), \rho \geq 0
$$

where $\mathrm{I}_{0}($.$) is the modified zeroth-order Bessel function of the first kind [see (24)] and M_{\omega_{k}}$ the non-centrality parameter of the class of change $\omega_{k}$ :

$$
M_{\omega_{k}}=\sqrt{\mu_{1, \omega_{k}}^{2}+\mu_{1, \omega_{k}}^{2}}
$$

It is worth noting that as $M_{\omega_{k}}$ becomes much larger than the standard deviation $\sigma_{\omega_{k}}$, then the Ricean distribution tends to become Gaussian.

Concerning the equation of the direction of the class of changed pixels $\omega_{k}$, it is possible to prove that, in the aforementioned assumptions, (26) can be simplified leading to the following Nonuniform distribution:

$$
\begin{aligned}
& p\left(\vartheta \mid \omega_{k}\right)=\exp \left(-\frac{\mu_{2, \omega_{k}}^{2}+\mu_{1, \omega_{k}}^{2}}{2 \sigma_{\omega_{k}}^{2}}\right)\left\{\frac{1}{2 \pi}+\frac{1}{2 \sigma_{\omega_{k}} \sqrt{2 \pi}} \frac{\mu_{2, \omega_{k}}^{2} \tan (\vartheta)+\mu_{1, \omega_{k}}^{2}}{\sqrt{\left(1+\tan ^{2}(\vartheta)\right)}}\right.
\end{aligned}
$$

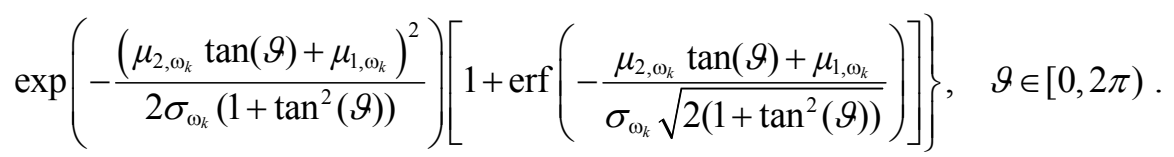

\section{Discussion}

In the previous sub-sections, we analyzed the statistical models more suitable to represent class

6 If the assumption is not verified, the general equations (23) and (26) should be used for modeling the statistical distributions of magnitude and direction, respectively, or a proper pre-processing should be applied to the data before using the simplified model (see section IV.C). 
distributions in the Polar domain in the general case and in some simplifying assumptions. Since the use of simplified models is of great importance for the development of effective and adequately complex automatic change-detection techniques, in this section we report a critical discussion on the assumptions considered for modeling the distributions of the classes of changed and unchanged pixels. In addition, we analyze practical implications of the theoretical analysis, in order to suggest criteria for driving the definition of proper pre-processing techniques for an effective data representation. Table I reports a summary of the theoretical statistical distributions derived (under simplifying assumptions) in subsections IV.A and IV.B for magnitude and direction of change and no-change classes.

TABLE I

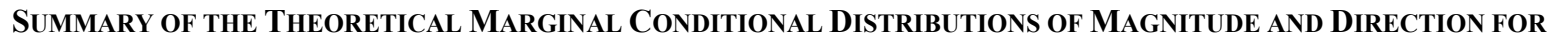 the Change and No-Change Classes Under Simplifying Assumptions.}

\begin{tabular}{c|c|c}
\hline \multirow{2}{*}{ Class } & \multicolumn{2}{|c}{ Conditional distribution } \\
\cline { 2 - 3 } & Magnitude $(\rho)$ & Direction $(\vartheta)$ \\
\hline Unchanged pixels $\left(\omega_{n}\right)$ & Rayleigh & Uniform \\
\hline Changed pixels $\left(\omega_{k}\right)$ & Rice & Non-Uniform \\
\hline
\end{tabular}

First of all, it is important to point out that a hypothesis at the basis of the theoretical analysis reported in the previous sub-sections consists in assuming independence among features describing SCVs in the Cartesian domain. The validity of this assumption depends on the considered images and applications, as well as on the investigated spectral channels. Significant deviations from this assumption affect the precision of the analytical distributions derived for describing the behaviors of changed and unchanged pixels. Nonetheless, if for a generic dataset the aforementioned assumption is not reasonable, it is possible to transform data from the original feature space to a transformed domain, in which features can be approximately modeled as independent. This can be obtained by applying a principal component transformation (PCT) to the features characterizing the SCVs [22]. In this way, at the cost of an additional transformation applied to the data, it is possible to properly adopt the analytical models described in sections IV.A and IV.B in the development of change- 
detection techniques $^{7}$.

The aforementioned assumption is at the basis of the presented theoretical analysis. All the other assumptions (discussed in the following) allow only to simplify the statistical distributions with respect to the general models in (23) and (26), which can be included in automatic techniques for operational change-detection algorithms, but are rather complex. For this reason, in the following we analyze the simplifying assumptions in greater detail and discuss possible preprocessing procedures aimed at transforming data so that these assumptions hold.

An important hypothesis that deserves to be discussed concerns the assumption that different features in the Cartesian SCV domain have similar standard deviations. The validity of the assumption in the original feature space depends on the considered images and applications, as well as on the investigated spectral channels. However, as for the assumption of the independence, if this approximation is not acceptable for the considered dataset, it is possible to transform the original feature space according to a procedure of diagonalization and whitening [22] and to apply changedetection algorithms to the transformed space.

A further relevant assumption to be analyzed for the class of unchanged pixels consists in the hypothesis that the mean vector components of the SCVs are equal to zero. This assumption is verified if the images are radiometrically corrected, so that the mean vectors are the same at the two dates (this condition can be always satisfied according to proper pre-processing strategies). Under this assumption we obtain that the magnitude has a Rayleigh distribution and the direction has a Uniform distribution. However, in some practical cases images are not radiometrically corrected and pre-processing procedures for matching the light conditions are neglected. According to the presented theoretical analysis, this may result in two main very critical effects: i) a possible increase of the overlapping of the classes of unchanged and changed pixels in the magnitude domain; ii) a strong deviation of the conditional distribution of the direction of unchanged pixels from the

It is worth noting that the PCT guarantees independence of features on the basis of the global distributions of patterns in the feature space. This means that after transformation the feature independence on the classes of 
expected Uniform model. The first effect is due to the fact that, although differences in light conditions result in a bias common to all classes in the Cartesian domain, when the non-linear magnitude operator is applied, the bias may result in an increase of overlapping between classes (this behavior will be shown in the experimental analysis reported in Section V.A). The second effect results from the observation that if the mean-value components of the SCVs are different from zero, the direction distribution of the class of unchanged pixels is no longer Uniform, but assumes a completely different behavior, which should be modeled with equation (35) (see Fig. 3). This has a dramatic impact on the data processing strategy, as it completely changes the distribution of the direction with respect to what expected in the ideal case. This observation confirms the importance of the radiometric correction step in the CVA technique, highlighting the critical effects of neglecting this step on the distributions of unchanged pixels.

Finally, another important implication derived from the theoretical analysis concerns the behavior of the distributions of the direction for the unchanged and changed classes, which are Uniform and Non-uniform, respectively. This means that it is possible to exploit the direction information (and in particular the modes associated to it for each changed class) for reducing the effects of the residual sources of noise present in the pre-processed multitemporal images (e.g. the registration noise, as it will be shown in the experimental analysis reported in Section V.A). This confirms from a formal theoretical point of view the analysis carried out in [4], where the direction information was used for identifying, modeling and reducing registration noise.

\section{EXPERIMENTAL RESULTS}

In order to assess the effectiveness of the proposed CVA framework, a number of experiments were carried out on a dataset made up of two multispectral images acquired by the Thematic Mapper (TM) multispectral sensor of the Landsat 5 satellite in the Island of Sardinia (Italy) in September $1995\left(t_{1}\right)$ and July $1996\left(t_{2}\right)$. The area selected for the experiments is a section $(412 \times 300$ pixels $)$ of 
the two scenes acquired by the TM sensor including Lake Mulargia. As an example of the images used in the experiments, Figs. 4 (a) and (b) show channel 4 of the September and July images, respectively.

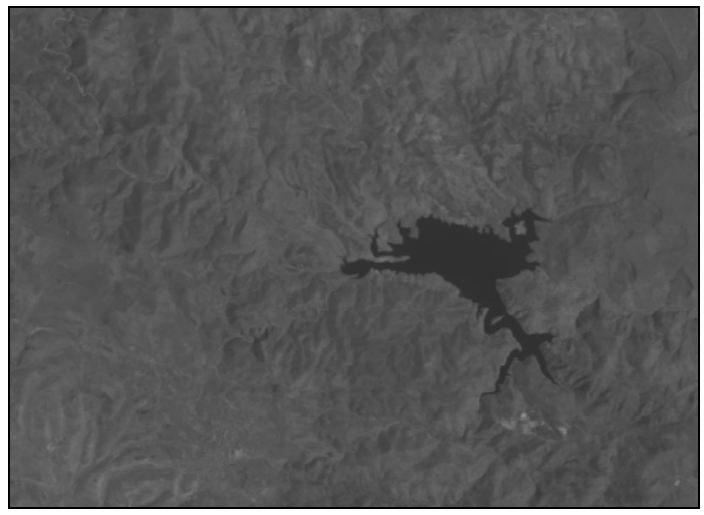

(a)

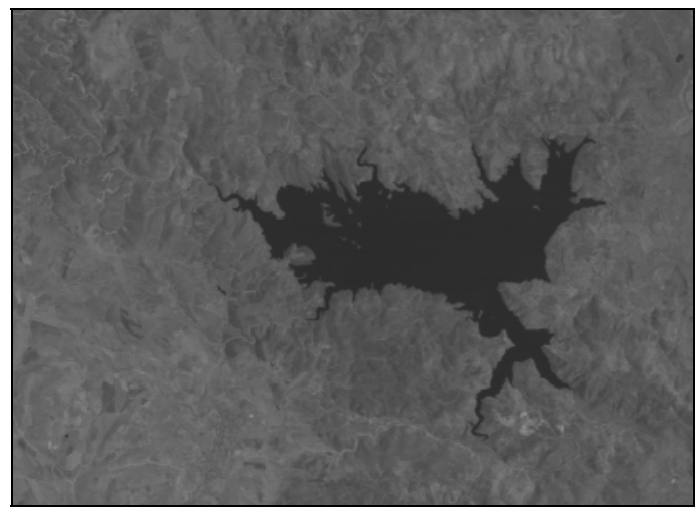

(b)

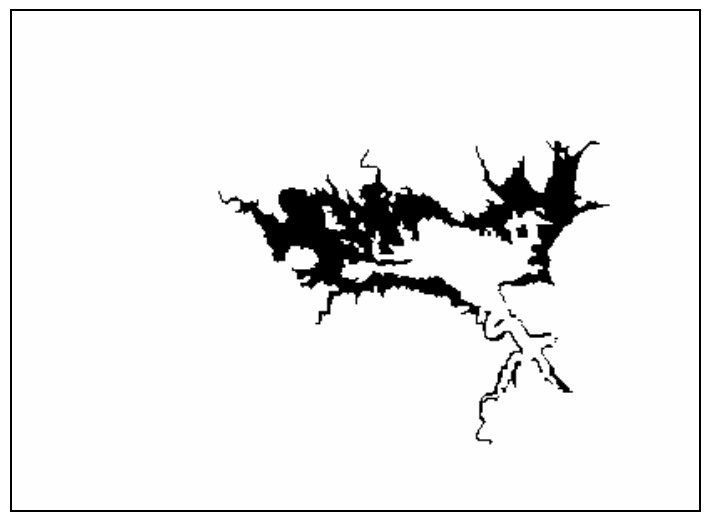

(c)

Figure 4 Images of the Lake Mulargia (Italy) used in the experiments. (a) Channel 4 of the Landsat-5 TM image acquired in September 1995; (b) Channel 4 of the Landsat-5 TM image acquired in July 1996; (c) available reference map of changed areas.

Between the two acquisition dates only one kind of change occurred in the investigated area which is related to the extension of water in the lake. The multitemporal images were coregistered and radiometrically corrected. A reference map of the analyzed site is available, where 7480 changed pixels and 116120 unchanged pixels are identified (see Fig. 4 (c)). This information is used for both computing the parameters of the statistical distributions for the classes of interest and evaluating the performances in terms of false and missed alarms of the change-detection process carried out by using the proposed statistical models.

The considered change-detection problem is relatively simple and thus it is suitable for a proper 
understanding of the properties and potentialities of the proposed framework. In this particular single-change problem, we define $\boldsymbol{\Omega}_{c}=\left\{\omega_{c_{1}}\right\}=\left\{\omega_{c}\right\}$. In the experiments, we considered only the two spectral channels 4 and 7 of the TM, i.e. the near and the middle infrared, as they are the most reliable for detecting changed areas. For simplicity of notation, in the following these channels will be referred with subscripts 1 and 2, respectively.

In order to assess the effectiveness of the proposed theoretical framework for analyzing and extracting SCV information, three different experiments have been carried out.

In the first experiment, a qualitative analysis of the true distributions of data in the Polar domain versus different pre-processing applied to the images is carried out. This experiment is aimed at pointing out the effects of the pre-processing procedures on both the data distributions and the precision of the models introduced in section IV for data representation.

The second experiment is aimed at validating the accuracy of the theoretical models of distributions presented in section IV in fitting the true data distributions for both the magnitude and the direction, under the simplifying assumptions introduced in sections IV.A and IV.B. Furthermore, the goodness-to-fit of the Rayleigh and Rice distributions adopted for the magnitude of the change and no-change classes, respectively, is compared with the goodness-to-fit of the widely used Gaussian model. Here, the well known Kolmogorov-Smirnov (KS) test is used for establishing whether a statistical model fits or not the true distribution [23]. The KS statistical test determines if two sets of data are drawn from the same statistical distribution. The test is based on the comparison between the cumulative distribution functions of the true data $S_{n}(x)^{8}$ (or empirical distribution function) and the expected one $F(x)$ (i.e., the cumulative distribution of the density function adopted for modeling the data) [23]. The KS test compares the cumulative distributions by means of a difference operator computing the so called KS-statistic $D_{n}$ :

$$
D_{n}=\sup _{x}\left\{F(x)-S_{n}(x) \mid\right\}
$$

$8 \quad x$ are the values for which both the cumulative densities are known. 
It is worth noting that $D_{n}$ is a random variable, whose distribution does not depend upon $F(x)$, i.e. the KS test is non-parametric and distribution free. The output of the KS-test consists in the acceptance of the assumption that the true data distribution follows the selected model if $D_{n} \leq D_{n}^{\alpha}$ with a high probability $P_{K S}$; else, the hypothesis is rejected and the two distributions are considered different. $D_{n}^{\alpha}$ is the critical value that depends on both the desired confidence level $\alpha$ and the number of samples $n$ used for estimating the empirical distribution function ${ }^{9}$.

The third and last experiment is aimed at establishing the possible improvements on the accuracy of the change-detection process (in terms of false and missed alarms, as well as total errors) by adopting the derived theoretical statistical models (for approximating the magnitude distributions of change and no-change pixels) rather than the widely used Gaussian model. In addition, an analysis on the impact of a poor pre-processing phase on the change-detection accuracy is also reported.

\section{A. Qualitative analysis of the class distributions in the Polar domain}

The aim of this experiment is to qualitatively show the effects of an inaccurate pre-processing phase (in terms of radiometric differences and/or misregistration noise) on the statistical distributions of magnitude and direction in the Polar coordinate system. In order to accomplish this analysis, we analyze the statistical distributions of SCVs obtained by applying the CVA technique to multitemporal images in three different cases: i) radiometrically corrected and coregistered images; ii) coregistered images without radiometric corrections; and iii) radiometrically corrected images with a poor coregistration (a residual shift of 2 pixels was accepted in both vertical and horizontal directions).

As expected from the theoretical analysis, in all cases it is possible to identify two clusters in the Polar domain. In the case of corrected images (Fig. 5 (a)), the first cluster is centered in the origin of the polar plot and shows high occurrences (red color) close to zero and a Uniform

9 Numerical values of $D_{n}^{\alpha}$ for different combination of $\alpha$ and $n$ are well known tabulated values [23]. 
distribution with respect to the direction domain. This cluster is associated to the unchanged SCVs. The second cluster shows a preferred direction and is located relatively far from the origin. This cluster is related to the SCVs associated with changed pixels. In this case, it is quite easy from a qualitative viewpoint to identify the decision boundary (threshold value on the magnitude) between the circle $C_{n}$ of no-changed pixels and the annulus $A_{c}$ of changed pixels. Furthermore, also the sector $S$ of the changed pixels is clearly visible (see Fig. 5 (a)).

The situation is significantly different in the second case, i.e. if no radiometric corrections are applied to the original images. Radiometric differences between the two acquisitions (see Fig. 5 (b)) have a dramatic impact on the distribution of the no-change class. As one can see, the cluster of unchanged pixels is no longer centered in zero; thus, the direction distribution is no further Uniform but assumes values in a subset of the domain, which is defined by the difference of the mean values of unchanged pixels at the two dates in the two considered spectral bands. In greater detail, in this condition, the no-change class distribution with non-zero mean can be approximated with the model described in section IV.B for the class of changed pixels. This behavior points out that the use of the Uniform model for the approximation of the distribution of the direction of the class of unchanged pixels in the data analysis phase (when images are not radiometrically corrected) is not acceptable and may result in poor performances. Furthermore, by analyzing Fig. 5 (b), it is possible to observe that the mean value of the magnitude of the unchanged pixels increases (with respect to the case of radiometrically corrected images), while the mean value of the magnitude of the changed pixels decreases. This means that if only the magnitude is used for the change detection (like in many real applications) the classes result more overlapped. This effect, which is due to the non-linearity of the magnitude operator, involves a higher change-detection error with respect to the case of radiometrically corrected data. In other words, the absence of radiometric corrections does not result in a bias contribution common to both classes, but may decrease significantly the separability between them in the magnitude domain. 


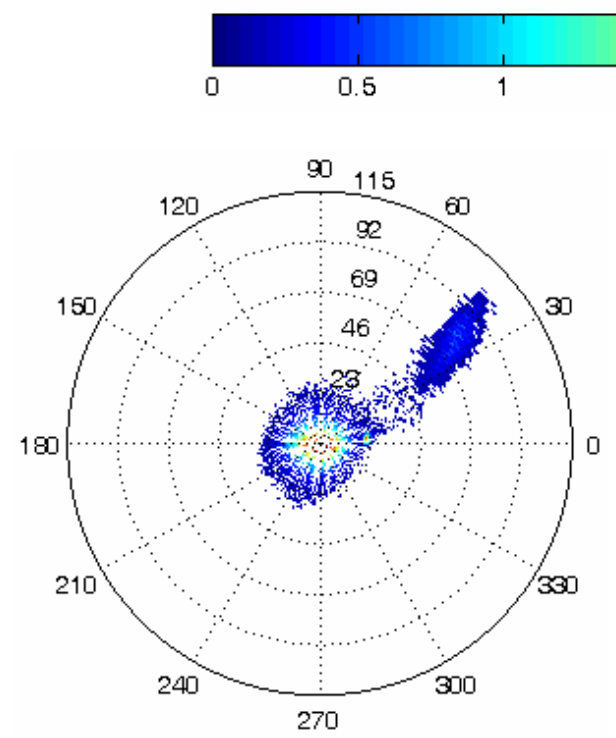

(a)

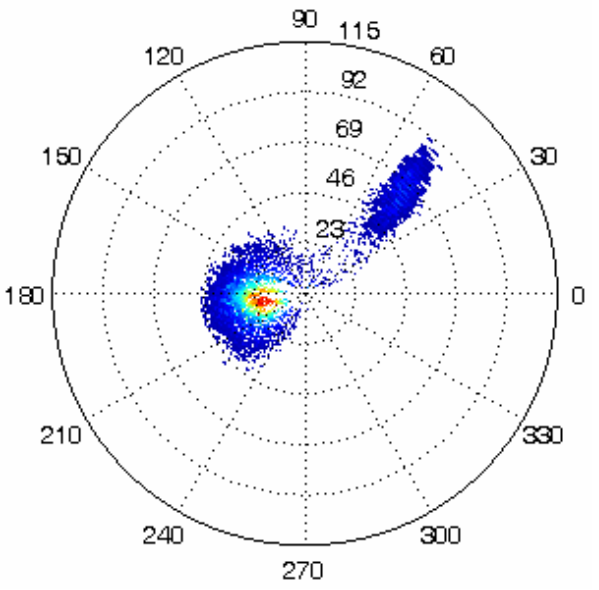

(b)

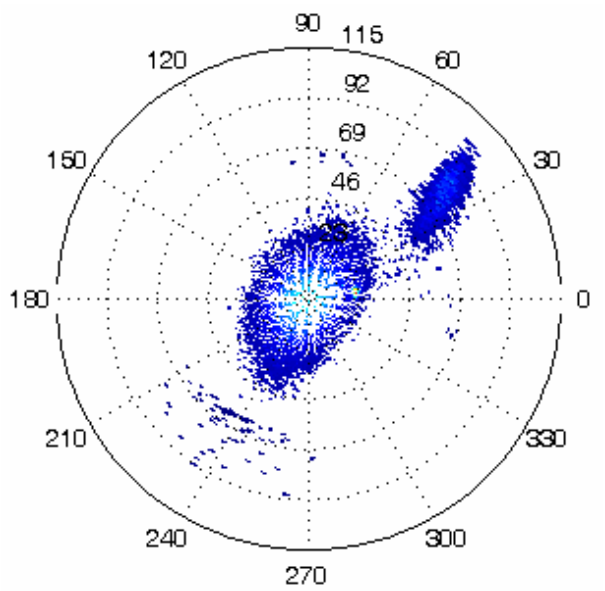

(c)

Figure 5 Histograms in the Polar coordinate system obtained after applying CVA to (a) multitemporal radiometrically corrected and coregistered images; (b) coregistered multitemporal images without radiometric corrections, (c) multitemporal radiometrically corrected images with a significant residual registration noise.

In the third case, image misregistration generates in the histogram plotted in the Polar domain: i) more spread $\mathrm{SCV}$ distributions; ii) the presence of unchanged SCVs that are out of $C_{n}$ and assume values in the entire direction domain. The spread increment is related to the non-perfect correspondence between multitemporal pixels, which leads to an increase of the variances of classes. The presence of pixels outside $C_{n}$ and $S$ is mainly due to the effects induced from border regions and details, which lead to the comparison of pixels belonging to completely different classes. These pixels have high magnitude values but direction that may differ from those of true 
changed pixels (Fig. 5 (c)). This behavior points out a very important guideline for practical applications, i.e. in situations where the residual misregistration between images can not be neglected, the use of the direction variable in addition to the magnitude one can reduce false alarms due to registration noise.

On the basis of the aforementioned analysis, it is clear that the Polar representation results in a useful qualitative tool for easily determining if a given dataset needs a pre-processing phase and which pre-processing steps (radiometric corrections, coregistration, etc.) should be applied for a proper data analysis.

B. Quantitative analysis of the accuracy of the statistical models of class distributions in the Polar domain

This experiment aims at a quantitative validation of the analytical models defined for approximating the statistical distributions of the magnitude and direction of the classes of changed and no-changed pixels. The validation is carried out according to the KS test. In these trials only the radiometrically corrected and coregistered images were considered.

In order to perform the validation of the derived statistical models, the true mean values and standard deviations of the change and no-change distributions were computed from $X_{D}$ in the Cartesian coordinate system on the basis of the available reference map. The obtained values are summarized in Tabs. 2 and 3. For the magnitude of the change and no-change classes also a comparison between the goodness-to-fit of Rice or Rayleigh model, respectively, and the commonly used Gaussian model is performed. In the following, the analysis of the results obtained on the two classes are considered separately.

\section{B.1. Statistical models for the class of no-changed pixels}

In order to adopt statistical models in (29) and (30) for the magnitude and the direction of unchanged SCVs, respectively, it should be verified if the hypotheses in (27) and (28) hold. By observing numerical values of standard deviations $\sigma_{b, \omega_{n}}$ in Tab. 2, it is reasonable to conclude that they are very similar to each other and can be approximated to the mean of the standard deviations 
(which is 9.49) thus satisfying (28). For the mean values no approximations should be introduced as (27) is verified (thanks to the use of the radiometrically corrected images).

TABLE 2

Mean Values and Standard Deviations for the Class of No-Changed PiXels in the Cartesian COORDINATE SYSTEM

\begin{tabular}{c|c|c}
\hline $\boldsymbol{b}$ & $\mu_{b, \omega_{n}}$ & $\sigma_{b, \omega_{n}}$ \\
\hline $\mathbf{1}$ (TM4) & 0 & 10.26 \\
\hline $\mathbf{2}$ (TM7) & 0 & 8.73 \\
\hline
\end{tabular}

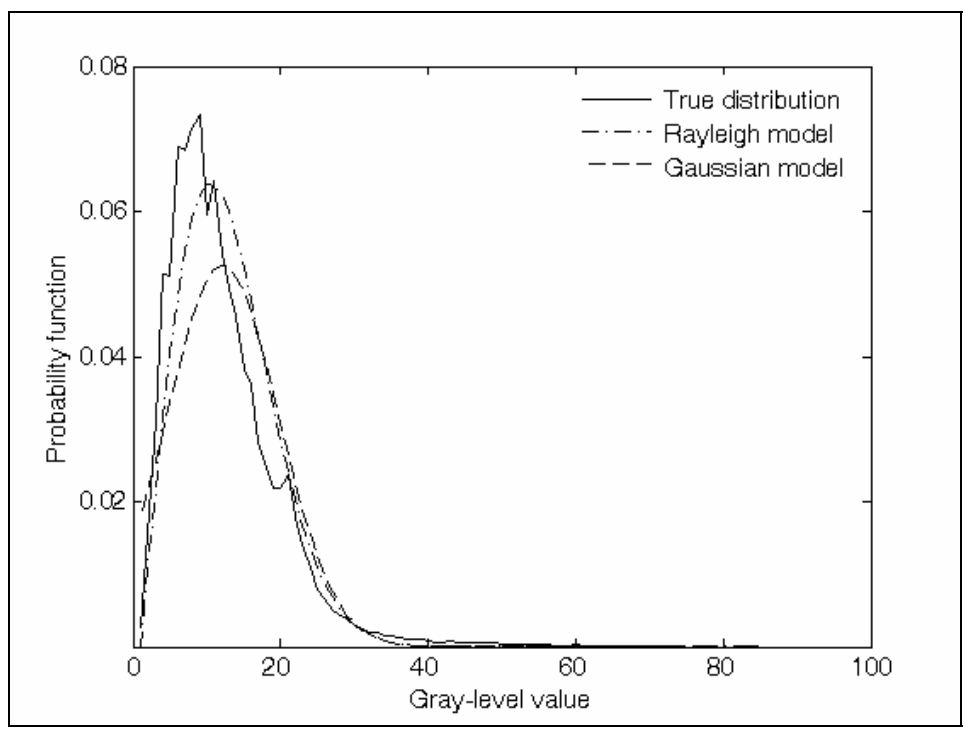

Figure 6 Comparison between the true behavior and the Gaussian and Rayleigh models for approximating the distribution of the magnitude of the unchanged pixels.

Let us first consider the magnitude variable. In Fig. 6 it is possible to see that the Rayleigh model approximates with good accuracy the distribution of the unchanged pixels (extracted from the reference map). In greater detail, this model fits better the data than the Gaussian model. This is confirmed by the KS test that results in a significantly higher $P_{K S}$ value for the Rayleigh model than for the Gaussian one (0.6396 vs. $\left.2 \cdot 10^{-4}\right)$. 


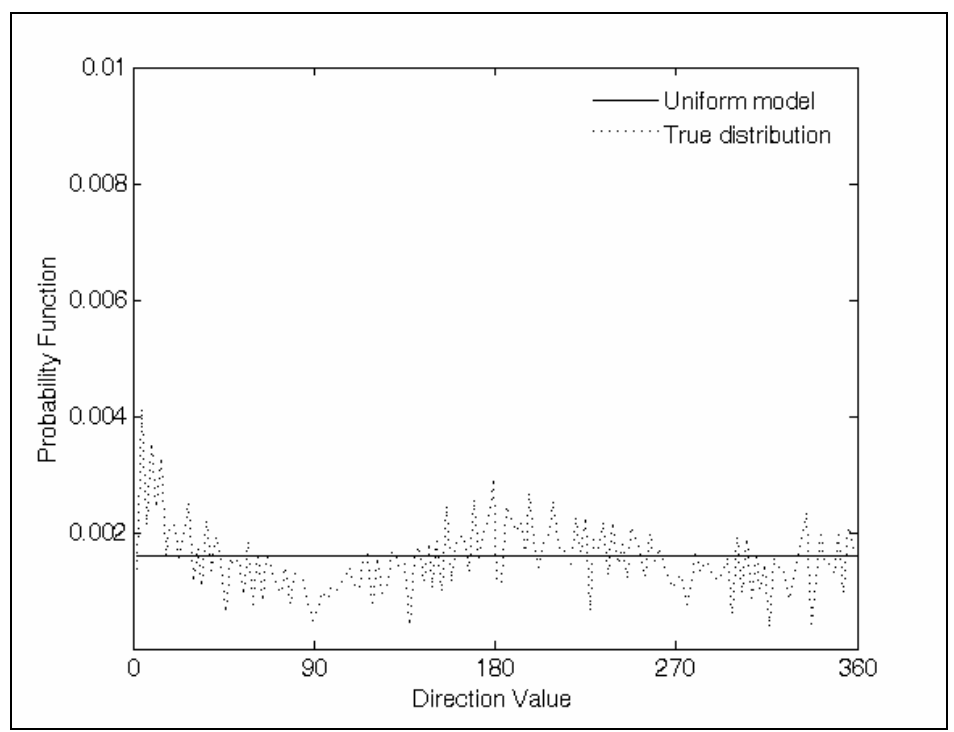

Figure 7 Comparison between the true behavior and the Uniform model for approximating the distribution of the direction of the unchanged pixels.

Let us now consider the behaviors of the distributions in the direction dimension. The KS test states that the SCV directions are uniformly distributed with a $P_{K S}$ value equal to 0.9939 . This result is confirmed also by a qualitative visual comparison between the true data distribution and the Uniform distribution (Fig. 7).

\section{B.2. Statistical models for the class of changed pixels}

In order to adopt statistical models in (33) and (35) for the magnitude and the direction of changed SCVs, respectively, it should be verified if the hypothesis in (32) hold. Similarly to the nochange class, from numerical values in Tab. 3 it is possible to observe that it is reasonable to approximate the standard deviation values $\sigma_{b, \omega_{c}}$ to the mean of the standard deviations, i.e. 9.77. This condition satisfies (32).

From Fig. 8 it is possible to see that the Rice model fits well the data in general, and slightly better than the Gaussian model. This is confirmed by the KS test that results in a slightly higher $P_{K S}$ value for the Rice model than for the Gaussian one ( 0.9993 vs. 0.9961$)$. The small difference in the two statistical models for this dataset is due to the fact that as the non-centrality parameter (34) becomes much larger than the standard deviation, then the Ricean distribution tends to become 


\section{Gaussian.}

TABLE 3

Mean Values and Standard Deviations Values for the Class of Changed Pixels in the Cartesian COORDINATE SYSTEM

\begin{tabular}{c|c|c}
\hline $\boldsymbol{b}$ & $\mu_{b, \omega_{c}}$ & $\sigma_{b, \omega_{c}}$ \\
\hline $\mathbf{1}$ (TM4) & 58.94 & 8.90 \\
\hline $\mathbf{2}$ (TM7) & 43.47 & 10.67 \\
\hline
\end{tabular}

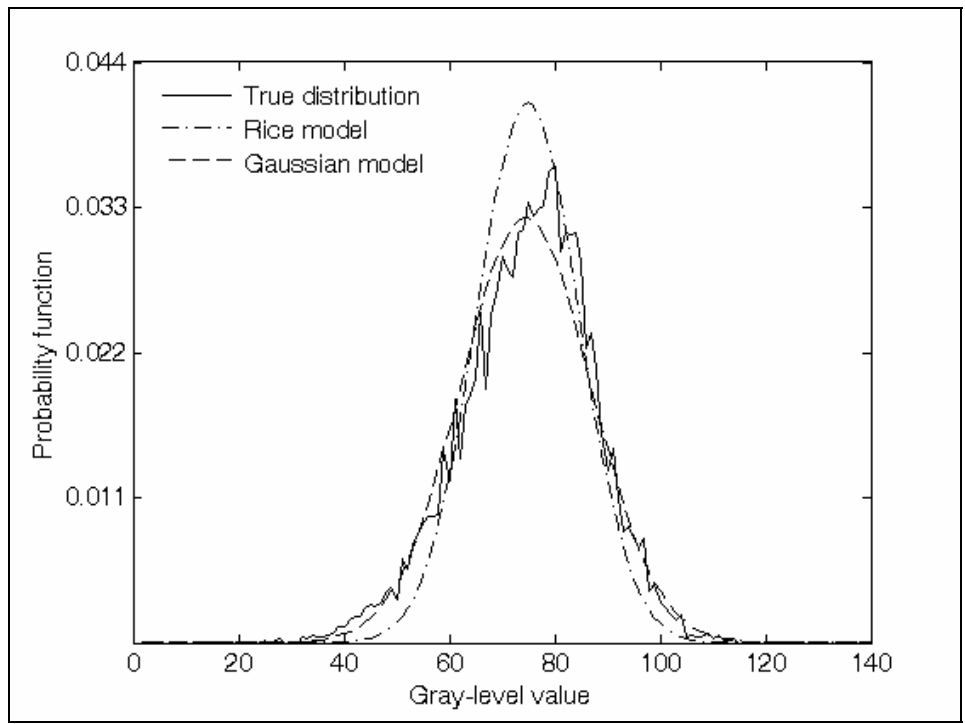

Figure 8 Comparison between the true behavior and the Gaussian and Rice models for approximating the distribution of the magnitude of the changed pixels.

The KS test states that the SCV direction is distributed according to (35) with a $P_{K S}$ value equal to 0.3306 . The relatively small value of $P_{K S}$ is due to the presence of SCVs whose direction differs from the expected one (see Fig. 9), as SCV direction is highly sensitive to noise components. Such outliers can be related to the presence of residual misregistration noise. 


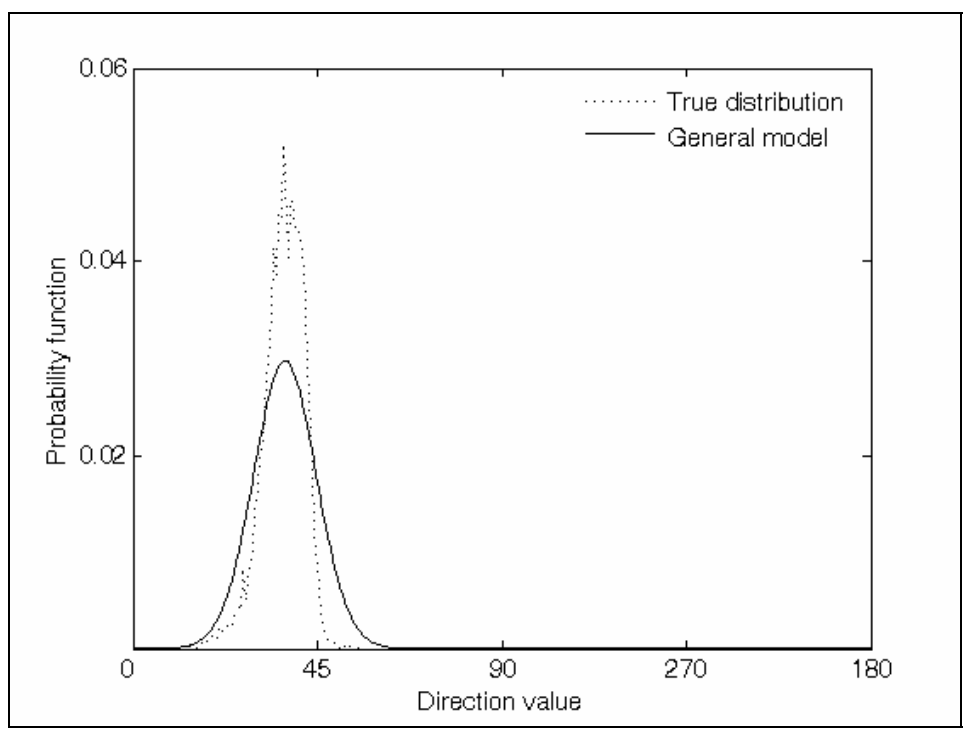

Figure 9 Comparison between the true behavior and the general Non-uniform model for approximating the distribution of the direction of the changed pixels.

C. Analysis of the effectiveness of the proposed framework for solving change-detection problems

This experimental part has two goals: i) the first is to evaluate the impact of radiometric correction on the performances of CVA; and ii) the second is to assess the improvement of the change-detection accuracy obtained by adopting the proposed statistical models rather than the Gaussian one for the change and no change classes in the magnitude domain.

In order to evaluate the impact of the radiometric corrections on the performances of the CVA technique, we have compared the accuracies yielded by thresholding the magnitude variable in the case of: i) radiometrically corrected and coregistered images; and ii) coregistered original images without radiometric corrections. To this end, the threshold values were defined according to a supervised trial-and-error procedure (MTEP), i.e. the minimum-error threshold was derived by performing a non-automatic evaluation of the overall change-detection errors versus all the possible values of the decision threshold; then the threshold value that yielded the minimum overall error was chosen. From the qualitative analysis carried out in subsection V.A, we expect that the changedetection accuracy is lower when no radiometric corrections are applied. As can be seen from Tab. 4, the MTEP procedure applied to the magnitude of the original dataset resulted in 1803 errors, 
while we obtain only 704 errors when thresholding is applied to the magnitude obtained after a very simple radiometric correction procedure which adjusted the mean value of the images. The overall error is more than halved. In greater detail, after rediometrically correcting the multitemporal images, both missed and false alarms decreased significantly from 529 to 369 pixels and from 1274 to 335 pixels, respectively.

\section{TABLE 4}

Overall Error, False Alarms and Missed Alarms (In Number of Pixels) ANd Threshold Value

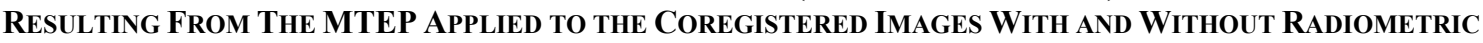
CORRECTIONS

\begin{tabular}{c|c|c|c|c}
\hline Radiometric corrections & $\begin{array}{c}\text { False } \\
\text { alarms }\end{array}$ & $\begin{array}{c}\text { Missed } \\
\text { alarms }\end{array}$ & $\begin{array}{c}\text { Overall } \\
\text { errors }\end{array}$ & $\begin{array}{c}\text { Threshold } \\
\text { value (T) }\end{array}$ \\
\hline None & 529 & 1274 & 1803 & 47 \\
\hline Mean adjustment & 369 & 335 & 704 & 51 \\
\hline
\end{tabular}

In order to asses the effectiveness of the proposed statistical models in solving change-detection problems, we considered only the coregistered and radiometrically corrected multitemporal dataset. Here, the MTEP results have been compared with the performances obtained by solving the changedetection problem according to the Bayes rule for minimum error (BRME) [22] under two different assumptions on statistical distributions: i) the proposed statistical models (i.e. Rayleigh model for the class of unchanged pixels and Rice model for the class of changed pixels); and ii) the widely used Guassian model for both classes.

As expected, thanks to the capability of the Rayleigh and Rice density functions to better model the true data distributions (see subsection V.B), the proposed models allow to obtain a lower amount of total errors with respect to the model based on the Gaussian distribution (956 vs. 1143). This is due to the fact that using the proposed model, the obtained threshold value (i.e., 43) is much closer to the optimal one (i.e., 51) than the threshold computed with the Gaussian model (i.e., 40). It is worth noting that although significant, the difference of threshold values between the Gaussian and the proposed models is relatively small. This depends on the fact that on the considered dataset the Rice distribution is close to the Gaussian one, as the non-centrality parameter is much larger then the standard deviation. We expect that higher improvements can be obtained in more general cases. 
TABLE 5

Overall Error, FAlse Alarms and Missed Alarms Resulting From The Selection Of THE DeCiSion Threshold Values Carried Out by Using MTEP, ANd BME with the Proposed Statistical Models and THE STANDARD GaUsSian Statistical MOdeL

\begin{tabular}{c|c|c|c|c}
\cline { 2 - 5 } & $\begin{array}{c}\text { False } \\
\text { alarms }\end{array}$ & $\begin{array}{c}\text { Missed } \\
\text { alarms }\end{array}$ & $\begin{array}{c}\text { Overall } \\
\text { errors }\end{array}$ & $\begin{array}{c}\text { Threshold } \\
\text { value (T) }\end{array}$ \\
\hline MTEP & 369 & 335 & 704 & 51 \\
\hline BME proposed models & 855 & 101 & 956 & 43 \\
\hline BME Gaussian model & 1086 & 57 & 1143 & 40 \\
\hline
\end{tabular}

\section{CONCLUSIONS}

In this paper, a Polar framework for a formal representation and definition of the change vector analysis (CVA) technique has been presented, as well as a related theoretical study on data distributions. The main motivation of this work relies on the observation that the CVA is a widely used technique for unsupervised change detection in multispectral and multitemporal remote sensing images, but a precise theoretically framework concerning its definition and use has not proposed in the literature (in many applications CVA in used without a proper understanding of the implications of the representation of the change information in the magnitude-direction domain). In this work, we aimed at filling this gap by introducing: i) a proper Polar framework for the representation and the analysis of multitemporal data in the context of the CVA technique; ii) a set of formal definitions (which are linked to the properties of the data) related to pattern representation in the Polar domain; iii) a theoretical analysis of the distributions of changed and unchanged pixels in the Polar domain; iv) a critical analysis of the theoretical study of distributions aimed at driving a proper exploitation of the information present in the Polar representation; v) an example of use of the proposed framework in a real change-detection problem, with a qualitative and quantitative evaluation of the reliability of the derived distributions and of the simplifying assumptions considered in the theoretical analysis.

In the light of the aforementioned contributions, we expect that the main impact of this work in the remote sensing community can be focused on the following issues: 
i) possibility to use in all practical applications of the CVA technique (irrespectively of the specific change-detection problem considered) a uniform polar representation with proper formal definitions of the different regions of interest based on the proposed framework;

ii) better understanding of the statistical properties of SCVs in the Polar domain and of the impact of the simplifying assumptions usually considered in the literature in the development of automatic data analysis algorithms;

iii) presentation of a solid background for the development of advanced and precise automatic algorithms for change detection, which properly takes into account the statistical properties of data in the Polar domain;

iv) better understanding of the fundamental role played by a proper pre-processing step (e.g., radiometric corrections, coregistration, PCT, diagonalization and whitening) for driving a correct design and use of efficient automatic data processing algorithms.

The analysis carried out points out that some of the simplifying assumptions usually adopted for representing data distributions in the Polar domain can become critical if a precise modeling of the change-detection problem is desired. Among the other properties discussed in the Section IV.C, we stress three observations:

- The theoretical and experimental analyses confirm that it may be critical solving the changedetection problems by representing the magnitude of classes of unchanged and changed pixels with Gaussian distributions, rather than using the more accurate models described in this paper (i.e. the Rayleigh distribution for unchanged pixels and the Rice distribution for changed pixels);

- radiometric corrections play a fundamental role in unsupervised change detection based on CVA: i) for increasing the separability between the classes of changed and unchanged pixels (by increasing the distance between the mean values of the two classes on the magnitude domain), and ii) for properly exploiting the direction information in the data processing phase (e.g., for identifying different kinds of change). If the radiometric corrections are neglected, the 
direction distribution of the class of unchanged pixels is completely different from the expected Uniform model, resulting in an important source of errors in the design of automatic dataprocessing techniques;

- the use of the direction information in the change-detection algorithms can be very important for reducing the false alarms induced from registration noise.

The effectiveness of the proposed Polar framework and of the related statistical analysis, as well as the importance of their implications have been verified on a real change-detection problem, by analyzing qualitatively and quantitatively the reliability of the simplifying assumptions considered in the theoretical analysis of data distributions and their impact on the precision of the models. The results obtained confirm that the theoretical models presented in this paper are suitable for a proper representation of the considered dataset when the CVA technique is adopted. In particular, these models significantly improve the representation of the distribution of data usually carried out with the reasonable, but in some cases imprecise, Gaussian model.

As future developments of this work, we are: i) studying the reformulation of threshold selection algorithms developed in the literature [15],[16] according to the distributions derived from the theoretical analysis reported in this paper; ii) considering the properties of the direction information for: a) devising effective unsupervised change-detection algorithms capable to automatically identify different kinds of change in a generic multitemporal dataset; b) better reducing the effects of the registration noise in the Polar domain.

\section{REFERENCES}

[1] A. Singh, "Digital change detection techniques using remotely-sensed data." Int. J. Remote sensing, vol. 10, no. 6, pp.989-1003, 1989.

[2] P.R. Coppin, I. Jonckheere and K. Nachaerts, "Digital change detection in ecosystem monitoring: A review," Int. J. Remote Sens., vol. 25, no. 9, pp. 1565-1596, May 2004. 
[3] D. Lu, P. Mausel, E. Brondízio and E. Moran, "Change detection techniques," Int. J. Remote Sens., vol. 25, no. 12, pp. 2365-2407, Jun. 2004.

[4] L. Bruzzone and R. Cossu, "Adaptive approach to reducing registration noise effects," IEEE Trans. Geosci. Rem. Sens., vol. 41, no. 11, pp. 2455-2465, Nov. 2003.

[5] W.A. Malila, "Change vector analysis: an approach for detecting forest changes with Landsat," Proc. LARS Machine Processing of Remotely Sensed Data Symposium, W. Lafayette, IN: Laboratory for the Application of Remote Sensing, pp. 326-336, 1980.

[6] J.L. Michalek, T.W. Wagner, J.J. Luczkovich and R.W. Stoffle, "Multispectral change vector analysis for monitoring coastal marine environments," Photogramm. Eng. Remote Sensing, vol. 59, no. 3, pp. 381-384, 1993.

[7] L.A. Virag and J.E. Colwell, “An improved procedure for analysis of change in Thematic Mapper image-pairs," Proc. of the XXI Int. Symposium of Remote Sensing of the Environment, Ann Arbor, MI, pp. 1101-1110, 26-30 October 1987.

[8] J.R. Jensen, Introductory Digital Image Processing: A Remote Sensing Perspective, Second Edition, Prentice Hall, Upper Saddle River, New Jersey, 1996.

[9] R.D. Johnson and E.S. Kasischke, "Change vector analysis: a technique for multispectral monitoring of land cover and condition," Int. J. Remote Sensing, vol. 19, no. 3, pp. 411-426, 1998.

[10] T.R. Allen and J.A. Kupfer, "Application of spherical statistics to change vector analysis of Landsat data: southern Appalachian spruce-fir forests," Remote Sensing Environment, vol, 74, pp. 482-493, 2000.

[11] J. Chen, P. Gong, C. He, R. Pu and P. Shi, "Land-use/land-cover change detection using improved change-vector analysis," Photogramm. Eng. Remote Sensing, vol. 69, no. 4, pp. 369-379, 2003.

[12] T. Warner, "Hyperspherical direction cosine change vector analysis," Int. J. Remote Sensing, vol. 26, no. 6, pp. 1201-1215, 2005. 
[13] K. Nackarets, K. Vaesen, B. Muys and P. Coppin, "Comparative performance of a modified change vector analysis in forest change detection," Int. J. Remote Sensing, vol. 26, no. 5, pp. 839-852, 2005.

[14] E.M. Pereira e A. W. Stezer, "Spectral characteristic of fire scars in Landsat-5 TM images of Amazonia”, Int. J. Remote Sensing, vol. 14, no. 11, 1993.

[15] L. Bruzzone, D. Fernández Prieto, “A minimum-cost thresholding technique for unsupervised change detection", Int. J. Remote Sensing, vol. 21, no. 18, pp. 3539-3544, Dec. 2000.

[16] L. Bruzzone, D. Fernández Prieto, "Automatic analysis of the difference image for unsupervised change detection", IEEE Trans. Geosci. Remote Sensing, vol. 38, no. 3, pp.1170-1182, 2000.

[17] J.A. Richards and X. Jia, Remote Sensing Digital Image Analysis: an Introduction, SpringerVerlag, New York, 1999.

[18] P. Beckmann, Probability in Communication Engineering, Harcourt, Brace \& World, INC., New York, 1967.

[19] J.R.G. Townshend, C.O. Justice and C. Gurney, "The impact of misregistration on change detection,” IEEE Trans. Geosci. Remote Sensing, vol. 30, pp. 1054-1060, Sept. 1992.

[20] L. Bruzzone and S.B. Serpico, "Detection of changes in remotely-sensed images by the selective use of multi-spectral information,” Int. J. Remote Sensing, vol. 18, no. 18, pp. 3883$3888,1997$.

[21] P.S. Chavez, Jr., "Radiometric calibration of Landsat Thematic Mapper multispectral images," Photogramm. Eng. Remote Sensing, vol. 55, no. 9, pp. 1285-1294, 1989.

[22] K. Fukunaga, Introduction to statistical pattern recognition, Academic press, Boston, Massachusset, 1990.

[23] P.G. Hoel, S.C. Port and C.J. Stone, Introduction to Statistical Theory, Houghton Mifflin Company, Atlanta, 1971. 